\title{
Control of D-STATCOM During Unbalanced Grid Faults Based on DC Voltage Oscillations and Peak Current Limitations
}

\author{
Arash Khoshooei, Javad.S.Moghani, \\ Jafar Milimonfared \\ Amirkabir University of Technology \\ Tehran, Iran \\ Khoshooei@aut.ac.ir,Moghani@aut.ac.ir, \\ Monfred@aut.ac.ir
}

\author{
Alvaro Luna ${ }^{1}$, Ignacio Candela ${ }^{1}$, Pedro Rodriguez ${ }^{1,2}$ \\ 1. Technical University of Catalunya UPC \\ Barcelona, Spain \\ Luna@ee.upc.edu, candela@ee.upc.edu \\ 2. Abengoa Research \\ Seville, Spain \\ pedro.rodriguez@abengoa.com,prodriguez@ee.upc.edu
}

\begin{abstract}
The safe operation of grid connected power converters during abnormal condition is a key issue in order to guarantee its operation and to avoid undesired trips. In this paper different control methods for the operation of a D-STATCOM are evaluated, where the reference currents are determined in such a way that none of the phase currents goes over the limits, as well as the DC voltage fluctuations remain in safe operation limit. Therefore, the contribution of this paper lays on the combination of the DC voltage oscillations and the current limit control. As it is shown in the following, three different control strategies are evaluated. The amplitude of the oscillations which are superimposed on the DC voltage as well as peak amplitude of the phase currents are calculated for each, considering a generic imbalance in the network. The effectiveness of the presented control strategies are verified by simulating a D-STATCOM tied to an industrial distribution network. Moreover a scaled scenario has been reproduced experimentally which shows that the results cope well with the analytical equations and the simulation results.
\end{abstract}

Keywords-D-STATCOM; unbalaced operation; current control; DC voltage oscillations;

\section{INTRODUCTION}

Grid codes worldwide are becoming more restrictive day by day [1]. The massive installation of Distributed Generation Power Supplies (DGPS), based on power converters brings the opportunity of utilizing their unique features to introduce grid supporting functionalities, even under severe transient conditions, such as grid faults. Nowadays, when a grid fault occurs, grid connected power converters are required not only to remain connected to the grid but also they must reduce their active power delivery and increase the reactive power injection for supporting the grid. Numerous research works have reported different power control strategies for DGPS or shunt connected power electronics converters, like STATCOMs, for operating them under abnormal grid conditions[2]-[4]. Since most of the grid faults are unbalanced faults, several research works have done for injecting unbalanced reactive currents to boost the positive sequence voltage as well as minimizing the negative sequence component. In an unbalanced grid, the interaction between positive and negative sequences in the voltage and their counterparts in the injected current results, in most of the cases, in active power fluctuations and consequently DC link voltage oscillations. The effectiveness of STATCOMs to enhance the stability margin of a fixed speed wind power plants is presented in [5]. Different strategies for injecting a coordinated combination of positive and negative sequence currents are introduced in [6]-[8]. Regardless of the control strategy objective, a safe operation of STATCOM from the perspective of maximum instantaneous phase currents, as well as the maximum instantaneous over voltage of DC bus because of fluctuations is critically important. Surpassing either of the aforementioned limits would give rise to an undesired STATCOM tripping. Controlling the maximum phase current of a STATCOM encountering an unbalanced grid faults was introduced in [9]. The effects of unbalanced supplying voltage on a conventional controlled STATCOM and its effects on DC voltage oscillations is discussed in [10]. For a STATCOM responsible for the regulation of positive and negative sequence voltages, [11] proposes to use a single phase inverter, in series with a DC link capacitor for eliminating the DC voltage oscillations during the fault period. However, a control algorithm which considers both criteria, DC bus voltage oscillations limit as well as phase current limitation has not been studied in deep. Moreover, up to now little work has been done on the limitation of DC voltage of STATCOMs facing severe unbalanced situations.

In this work, three different reactive power injection strategies for controlling a STATCOM are analyzed, Average Active Reactive Control(AARC), Balanced Positive Sequence Control (BPSC) and Positive Negative Sequence Control(PNSC). As the current limitation in each has been previously studied, this paper is more focused on the deduction of the mathematical expressions for active power fluctuations and the associated DC voltage oscillations, in such a way that the maximum permissible reactive power reference can be determined. By comparing this reference and 
a previously calculated maximum reference value, which satisfies the current limitations, the final reactive power reference is chosen, which will respect both the DC voltage oscillation and peak current limitation.

The organization of the paper is as follows. Section II discusses the basics of the three different reactive power control strategies. The derivation of active power fluctuations and consequence DC voltage oscillations are presented in section III. Section IV is devoted to calculation of maximum phase currents. The overall D-STATCOM control system is discussed in section $\mathrm{V}$ and the performance of a D-STATCOM, connected to a weak industrial network experiencing fault condition is analyzed in section VI. Finally the experimental evaluation of a laboratory scaled D-STATCOM considering both limiting criteria is shown in section VII, just before the conclusions.

\section{Different Reactive Power Control Strategies}

In an arbitrary three phase network with unbalanced variables $f \in\{v, i\}$ and supposing a three wire system as well as the availability of a $\Delta$ connection in one of the windings of interfacing transformer, as shown in Fig. 1, the zero sequence voltages and currents at the point of connection of the converter to the grid will be eliminated.

Therefore, by using a constant amplitude Clark Transformation, we can write:

$$
\left[\begin{array}{l}
f_{\alpha}(t) \\
f_{\beta}(t)
\end{array}\right]=\left[\begin{array}{ccc}
2 / 3 & -1 / 3 & -1 / 3 \\
0 & 1 / \sqrt{3} & -1 / \sqrt{3}
\end{array}\right]\left[\begin{array}{c}
f_{a}(t) \\
f_{b}(t) \\
f_{c}(t)
\end{array}\right]
$$

where $f_{i}(t), i \in\{a, b, c\}$ are phase variables (voltages and currents), furthermore each variable in stationary reference frame can be decomposed into a couple of balanced sets of positive $(+)$ and negative $(-)$ variables as shown below:

$$
\begin{aligned}
& f_{\alpha}(t)=f_{\alpha}^{+}(t)+f_{\alpha}^{-}(t) \\
& f_{\beta}(t)=f_{\beta}^{+}(t)+f_{\beta}^{-}(t)
\end{aligned}
$$

In fact, it is very common to use a couple of in-quadrature $90^{\circ}$ shifted vectors to develop the reactive power definition:

$$
\begin{aligned}
& f_{\perp \alpha}(t)=f_{\perp \alpha}^{+}(t)-f_{\perp \alpha}^{-}(t) \\
& f_{\perp \beta}(t)=f_{\perp \beta}^{+}(t)-f_{\perp \beta}^{-}(t)
\end{aligned}
$$

Fig. 2 represents system variables in the stationary reference frame. According to Fig. 2, the time expressions for the

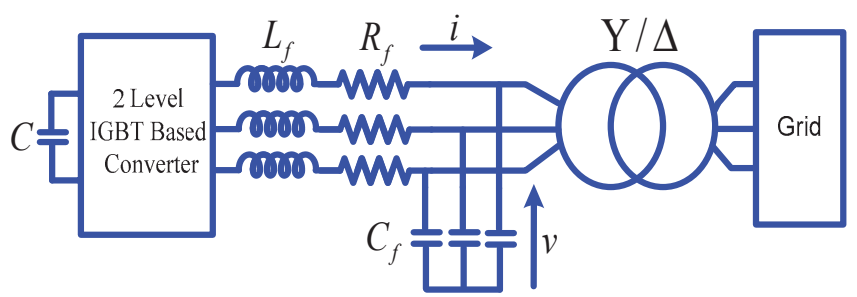

Fig .1. Structure of a D-STATCOM connected to the grid positive and the negative sequences for both the real and inquadrature variables can be written as:

$$
\begin{gathered}
f_{\alpha}^{+}(t)=F^{+} \cos \left(\omega t+\theta_{f}^{+}\right) \\
f_{\beta}^{+}(t)=F^{+} \cos \left(\omega t+\theta_{f}^{+}-\frac{\pi}{2}\right) \\
f_{\alpha}^{-}(t)=F^{-} \cos \left(-\omega t+\theta_{f}^{-}\right) \\
f_{\beta}^{-}(t)=F^{-} \cos \left(-\omega t+\theta_{f}^{-}-\frac{\pi}{2}\right) \\
f_{\perp \alpha}^{+}(t)=F^{+} \cos \left(\omega t+\theta_{f}^{+}-\frac{\pi}{2}\right) \\
f_{\perp \beta}^{+}(t)=F^{+} \cos \left(\omega t+\theta_{f}^{+}-\pi\right) \\
f_{\perp \alpha}^{-}(t)=F^{-} \cos \left(-\omega t+\theta_{f}^{-}-\frac{\pi}{2}\right) \\
f_{\perp \beta}^{-}(t)=F^{-} \cos \left(-\omega t+\theta_{f}^{-}-\pi\right)
\end{gathered}
$$

In case of using constant amplitude Clark Transformation, active and reactive powers can be written as:

$$
\begin{gathered}
p=\left(\frac{3}{2}\right) \mathbf{v} . \mathbf{i} \\
q=\left(\frac{3}{2}\right) \mathbf{v}_{\perp} . \mathbf{i}
\end{gathered}
$$

where $\mathbf{v}, \mathbf{v}_{\perp}$ and $\mathbf{i}$ are voltage, in-quadrature voltage and current vectors respectively.

Details of AARC, BPSC and PNCS schemes and their characteristics are given in [12] and the reference currents are shown in Table I. $P^{*}$ and $Q^{*}$ are active and reactive power set points and $V^{+}$and $V^{-}$are the voltage positive and negative sequence amplitudes respectively.

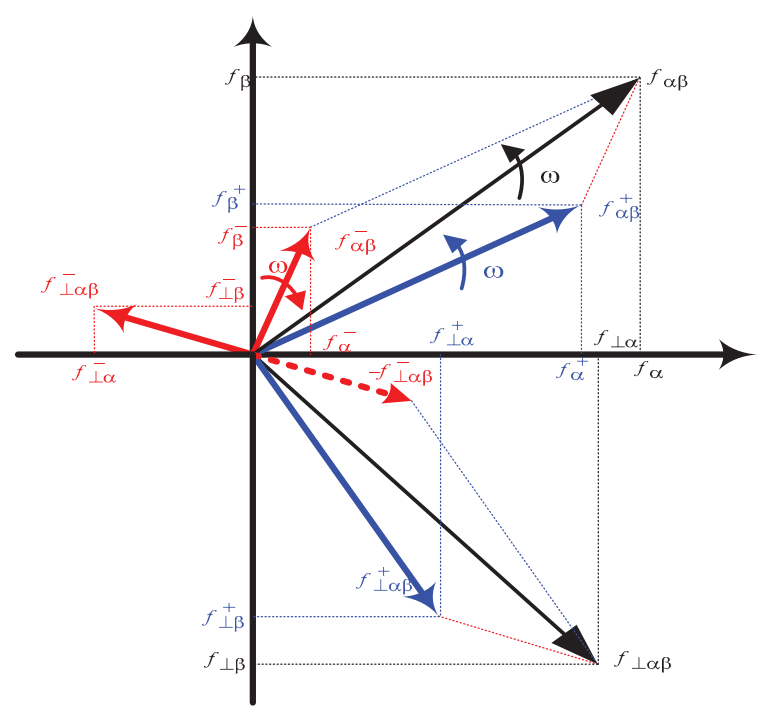

Fig. 2. Vector representation in stationary reference frame 
Table I. Reference current vectors for different power injection schemes

\begin{tabular}{|c|c|}
\hline Scheme & Reference Current Vector \\
\hline AARC & $\mathbf{i}^{*}=\frac{(2 / 3) P^{*}}{\left(V^{+}\right)^{2}+\left(V^{-}\right)^{2}} \mathbf{v}+\frac{(2 / 3) Q^{*}}{\left(V^{+}\right)^{2}+\left(V^{-}\right)^{2}} \mathbf{v}_{\perp}$ \\
\hline BPSC & $\mathbf{i}^{*}=\frac{(2 / 3) P^{*}}{\left(V^{+}\right)^{2}} \mathbf{v}^{+}+\frac{(2 / 3) Q^{*}}{\left(V^{+}\right)^{2}} \mathbf{v}_{\perp}^{+}$ \\
\hline PNSC & $\mathbf{i}^{*}=\frac{(2 / 3)}{\left(V^{+}\right)^{2}-\left(V^{-}\right)^{2}}\left[P^{*}\left(\mathbf{v}^{+}-\mathbf{v}^{-}\right)+Q^{*}\left(\mathbf{v}_{\perp}^{+}+\mathbf{v}_{\perp}^{-}\right)\right]$ \\
\hline
\end{tabular}

\section{EFFECT OF DIFFERENT REACTIVE POWER CONTROL} StRategies ON DC Bus Voltage Oscillations

This section is devoted to the calculation of active power fluctuations in the three aforementioned reactive power control strategies considering unbalanced voltage condition. Furthermore, a step by step derivation of the DC voltage oscillations, based on the principle of energy conservation is presented. Finally, some hints for proper DC capacitor selection are presented.

\section{A. Active Power Fluctuations}

According to the instantaneous power theory[13], the active power fluctuations at the terminal of a power converter could be written as:

$$
\tilde{p}(t)=(3 / 2)\left(v_{\alpha}^{+} i_{\alpha}^{-}+v_{\alpha}^{-} i_{\alpha}^{+}+v_{\beta}^{+} i_{\beta}^{-}+v_{\beta}^{-} i_{\beta}^{+}\right)
$$

For extracting the voltage sequence components used in (19), the main principles of several research works, such as [14] is considered.

It could be inferred from (19) that the active power fluctuation is a consequence of the different sequence voltages and currents interaction. In other words, for a balanced voltage and pure balanced positive sequence current injection, there is no power fluctuation. At the other extreme, when the voltage is almost balanced and the converter only injects a negative sequence current to the grid, the amplitude of the power fluctuations reaches its maximum value. This condition is very probable when the D-STATCOM works in a load current balancing mode. Under unbalanced grid fault conditions, when the D-STATCOM works in grid voltage supporting mode, positive sequence voltage is always higher than the negative sequence voltage, therefore, the strategies which inject more negative sequence current, produces higher active power fluctuations.

In (19) the current components are generated by the control block with respect to the reactive power injection scheme. Reference currents for each aforementioned strategy could be achieved by inserting the arbitrary voltage of (6) to(13) into (16)-(18). The D-STATCOM ohmic losses compared with its rated V.A is insignificant so the reference active power is almost zero $\left(P^{*} \approx 0\right)$. Inserting the calculated reference currents as well as the voltage components in (19), the active power fluctuations for different schemes are introduced in
Table II. Active power fluctuations for different schemes

\begin{tabular}{|c|c|}
\hline Scheme & Active Power Fluctuations \\
\hline AARC & $\tilde{p}(t)=0$ \\
\hline BPSC & $\tilde{p}(t)=\lambda \cdot Q^{*} \sin \left(2 \omega t+\theta_{v}^{+}-\theta_{v}^{-}\right)$ \\
\hline PNSC & $\tilde{p}(t)=\frac{2 Q^{*} \cdot \lambda}{1-\lambda^{2}} \sin \left(2 \omega t+\theta_{v}^{+}-\theta_{v}^{-}\right)$ \\
\hline
\end{tabular}

Table II, where $\lambda$ is the Voltage Unbalance Factor (VUF) as a measure of severity of voltage imbalance which is defined as:

$$
\lambda=V^{-} / V^{+}
$$

Regardless of the AARC scheme that presents no fluctuations in active power, two later schemes experience a second order component fluctuations with the amplitudes influenced from reactive power set points and the voltage unbalance factor.

\section{B. DC Capacitor Voltage Oscillations}

Neglecting the converter losses and according to the energy conservation theory, the DC link power absorption $\left(p_{c}(t)\right)$ is the same as the input power, therefore:

$$
p_{c}(t)=\tilde{p}(t)
$$

The DC link capacitor voltage is:

$$
v_{c}(t)=\bar{V}_{c}+\tilde{v}_{c}(t)
$$

where this voltage is a composition of a constant component $\left(\bar{V}_{c}\right)$ and a fluctuating component $\left(\tilde{v}_{c}(t)\right)$, as a result:

$$
p_{c}(t)=v_{c}(t) \cdot i_{c}(t)=v_{c}(t) \cdot C \frac{d v_{c}(t)}{d t}
$$

by substituting (25) in (26) :

$$
p_{c}(t)=C\left(\bar{V}_{c} \cdot \frac{d \tilde{v}_{c}(t)}{d t}+\tilde{v}_{c}(t) \cdot \frac{d \tilde{v}_{c}(t)}{d t}\right) \approx C \cdot \bar{V}_{c} \cdot \frac{d \tilde{v}_{c}(t)}{d t}
$$

in the above equation, the second term in comparison to the first one is negligible therefore, by integrating (27) an equation for the DC voltage oscillations is attained:

$$
\tilde{v}_{c}(t)=\frac{1}{C \cdot \bar{V}} \int p_{c}(t) d t=\frac{1}{C \cdot \bar{V}} \int \tilde{p}(t) d t
$$

DC voltage oscillations, proportionally relate to the active power fluctuations. In contrast, higher the DC voltage value or capacitance, lower is the DC voltage oscillations.

Using (20)-(22) in (28), a superimposed second order oscillations on the average DC value for all the aforementioned control schemes are listed in Table III. It is clear that the higher voltage unbalance factor, the higher is the DC voltage deviation. With a fixed modulation algorithm, high amount of oscillations superimposed on the DC voltage, introduce non-characteristics harmonics in the output voltage spectrum. The deviation above the average value is more important than the undergoing voltage. Overvoltage has detrimental effects on the semiconductor switches and the DC link capacitor, might actuate the DC over voltage protection unit. 
Table III. DC voltage oscillations for different schemes

\begin{tabular}{|c|c|}
\hline Scheme & DC Voltage Oscillations \\
\hline AARC & $\tilde{v}_{c}(t)=0$ \\
\hline BPSC & $\tilde{v}_{c}(t)=\frac{-Q^{*} \cdot \lambda}{2 \omega \cdot C \bar{V}_{c}} \cos \left(2 \omega t+\theta_{v}^{+}-\theta_{v}^{-}\right)$ \\
\hline PNSC & $\tilde{v}_{c}(t)=\frac{-Q^{*} \cdot \lambda}{\omega \cdot C \bar{V}_{c}\left(1-\lambda^{2}\right)} \cos \left(2 \omega t+\theta_{v}^{+}-\theta_{v}^{-}\right)$ \\
\hline
\end{tabular}

For a specified permissible DC overvoltage, the maximum reactive power can be determined. DC voltage oscillations amplitude for a typical 4MVA D-STATCOM, delivering rated V.A, with respect to the voltage unbalanced factor is presented in Fig. 3. The active power fluctuations is not occurred in AARC strategy and it is the finest strategy for preventing the DC voltage oscillations. On the other hand, PNSC strategy suffers from high DC voltage deviation in large VUFs and if the reactive power set-point is not reduced properly it might result in converter tripping.

\section{DC Capacitor Selection to Meet the Criteria}

The main criteria for DC capacitor sizing is to be sure about the STATCOM capability in the regulation of voltage during transients. Different research works have presented different methods for sizing the capacitor with regards to transient performance requirements [15]-[16]. However, fault ride through performance of the STATCOM and the effect of capacitor size on the DC voltage oscillations is not considered in previous works. The main principle for all the methods used for capacitor sizing lays on the fact that the change in the capacitor's stored energy should be equal to a multiplication of the STATCOM rated power $\left(S_{\text {rated }}\right)$ by a specified period of time, e.g. 0.5-1 cycle. A typical relation is :

$$
\frac{1}{2} C\left(\bar{V}_{c, \text { max }}^{2}-\bar{V}_{c, \text { min }}^{2}\right)=k_{s} \cdot S_{\text {rated }} \cdot T_{\text {tran }}
$$

where $\bar{V}_{c, \text { max }}$ and $\bar{V}_{c, \text { min }}$ are the maximum and the minimum permissible values for DC voltage. $k_{s}$ is a coefficient that determines the share of STATCOM contribution for a specific transient time, $T_{\text {tran }}$.

For limiting the amplitude of the DC voltage oscillations, a level of immunity could be defined like:

$$
\left|\tilde{v}_{c}(t)\right| \leq k \cdot \bar{V}_{c}
$$

where $\left|\tilde{v}_{c}(t)\right|$ is the amplitude of DC voltage oscillations and $k$ is the allowed percentage of nominal DC voltage.

In the AARC strategy, DC voltage oscillations are zero and the value of capacitance is derived from (32). By inserting the oscillations amplitude from (30) in (33), the minimum capacitance to meet DC voltage oscillations for BPSC is:

$$
C \geq \frac{Q^{*} \cdot \lambda}{2 \omega \cdot C \cdot k \cdot \bar{V}_{c}^{2}}
$$

In the same way by combining (31) and (33) for PNSC, the minimum capacitance value is calculated as:

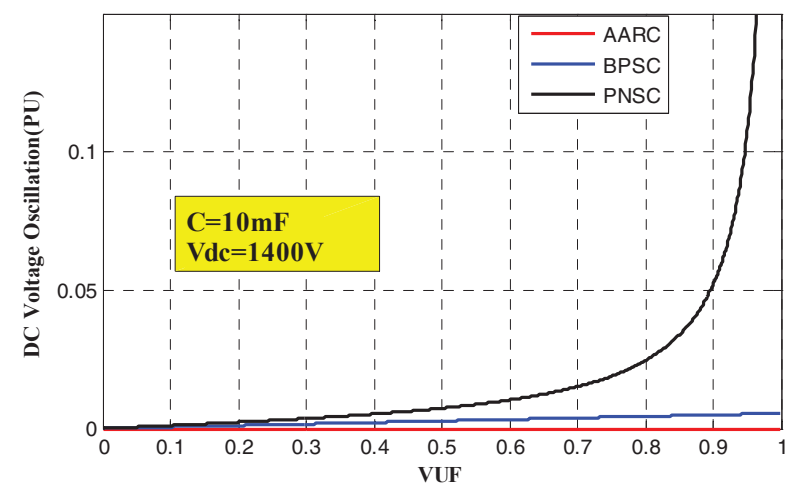

Fig. 3. DC voltage oscillations of a typical 4MVA D-STATCOM

$$
C \geq \frac{Q^{*} \cdot \lambda}{\omega \cdot C \cdot k \cdot \bar{V}_{c}^{2}\left(1-\lambda^{2}\right)}
$$

The maximum value of the calculated capacitance among (32) and (33), meets both the transient response requirement as well as limitation of DC voltage oscillations.

\section{MaXimum Phase CurRent in Different Reactive POWER CONTROL STRATEGIES}

Considering an unbalanced voltage condition, if the reactive power set point is not reduced, it is likely that currents in one or more phases pass over their nominal values and the over current protection of the converter would be activated. This section concentrates on the derivation of new reactive power set point for each strategy in which the maximum of phases currents kept in a safe region according to the nominal current. Choosing a set of arbitrary equation for phase currents in natural $(a b c)$ frame as:

$$
\left[\begin{array}{c}
i_{a}(t) \\
i_{b}(t) \\
i_{c}(t)
\end{array}\right]=\left[\begin{array}{c}
I_{a} \cos \left(\omega t+\varphi_{a}\right) \\
I_{b} \cos \left(\omega t+\varphi_{b}\right) \\
I_{c} \cos \left(\omega t+\varphi_{c}\right)
\end{array}\right]
$$

for each strategy the magnitude of maximum phase current according to the positive and negative sequence voltage components are extracted and then the permissible amount of reference reactive power is calculated.

\section{A. Maximum Phase Current for AARC Strategy}

Considering (16), the reference current for AARC is:

$$
\mathrm{i}^{*}=\left[\begin{array}{c}
i_{\alpha}^{*} \\
i_{\beta}^{*}
\end{array}\right]=b_{1} \cdot\left[\begin{array}{c}
v_{\perp \alpha} \\
v_{\perp \beta}
\end{array}\right]=b_{1} \cdot\left[\begin{array}{c}
v_{\beta} \\
-v_{\alpha}
\end{array}\right]=b_{1} \cdot\left[\begin{array}{c}
v_{\beta}^{+}+v_{\beta}^{-} \\
-v_{\alpha}^{+}-v_{\alpha}^{-}
\end{array}\right]
$$

where $b_{1}$ is an instantaneous susceptance and defined as:

$$
b_{1}=\frac{(2 / 3) Q^{*}}{\left(V^{+}\right)^{2}+\left(V^{-}\right)^{2}}
$$

putting the time domain positive and negative voltage components from (6) to (9) in (37), magnitude of maximum phase current are calculated as:

$$
\begin{gathered}
I_{a}=b_{1} \sqrt{\left(V^{+}\right)^{2}+\left(V^{-}\right)^{2}+2 V^{+} \cdot V^{-} \cos (\delta+\pi)} \\
I_{b}=b_{1} \sqrt{\left(V^{+}\right)^{2}+\left(V^{-}\right)^{2}+2 V^{+} \cdot V^{-} \cos (\delta-\pi / 3)}
\end{gathered}
$$




$$
I_{c}=b_{1} \sqrt{\left(V^{+}\right)^{2}+\left(V^{-}\right)^{2}+2 V^{+} \cdot V^{-} \cos (\delta+\pi / 3)}
$$

where $\delta=\theta_{v}^{+}+\theta_{v}^{-}$which is available at the output of sequence extraction block. The maximum safe amplitude of the phase currents is the nominal one. For a specific unbalanced condition the maximum permissible reactive power in which none of the phase currents surpass the limitation could be determined. By inserting (23) and (38) in (39) to (41), the maximum allowed reactive power as a function of positive sequence voltage and VUF could be obtained. This relation is presented in Fig. 4. It is clear that in case of faulty condition the reactive power set point must be decreased to maintain the phase current less than the rated values. It is worth mentioning that some of the point in this graph are not achievable in practice.

\section{B. Maximum Phase Current for BPSC Strategy}

The reference current for BPSC strategy is inspired from (17) and is expressed as:

$$
\mathrm{i}^{*}=\left[\begin{array}{c}
i_{\alpha}^{*} \\
i_{\beta}^{*}
\end{array}\right]=b_{2} \cdot\left[\begin{array}{c}
v_{\perp \alpha}^{+} \\
v_{\perp \beta}^{+}
\end{array}\right]=b_{2} \cdot\left[\begin{array}{c}
v_{\beta}^{+} \\
-v_{\alpha}^{+}
\end{array}\right]
$$

where $b_{2}$ is defined as:

$$
b_{2}=\frac{(2 / 3) Q^{*}}{\left(V^{+}\right)^{2}}
$$

In this strategy all the phases have same amplitude which is calculated as:

$$
I_{a}=I_{b}=I_{c}=\frac{(2 / 3) Q^{*}}{V^{+}}
$$

From (44) it could be inspired that for keeping the phase currents safely to rated value, the maximum reference reactive power must be reduced in proportion of $V^{+}$.

\section{Maximum Phase Current for PNSC Strategy}

According to (18), in PNSC strategy the current controller must track the following reference current:

$$
\mathrm{i}^{*}=\left[\begin{array}{c}
i_{\alpha}^{*} \\
i_{\beta}^{*}
\end{array}\right]=b_{3} \cdot\left[\begin{array}{c}
v_{\perp \alpha}^{+}+v_{\perp \alpha}^{-} \\
v_{\perp \beta}^{+}+v_{\perp \beta}^{-}
\end{array}\right]=b_{3} \cdot\left[\begin{array}{c}
v_{\beta}^{+}+v_{\beta}^{-} \\
-v_{\alpha}^{+}-v_{\alpha}^{-}
\end{array}\right]
$$

where $b_{3}$ is defined as:

$$
b_{3}=\frac{(2 / 3) Q^{*}}{\left(V^{+}\right)^{2}-\left(V^{-}\right)^{2}}
$$

By applying (6)-(9) in (45) and applying reverse Clark transformation, the phase current amplitudes are obtained as:

$$
\begin{gathered}
I_{a}=b_{3} \sqrt{\left(V^{+}\right)^{2}+\left(V^{-}\right)^{2}+2 V^{+} \cdot V^{-} \cos (\delta)} \\
I_{b}=b_{3} \sqrt{\left(V^{+}\right)^{2}+\left(V^{-}\right)^{2}+2 V^{+} \cdot V^{-} \cos (\delta+2 \pi / 3)} \\
I_{c}=b_{3} \sqrt{\left(V^{+}\right)^{2}+\left(V^{-}\right)^{2}+2 V^{+} \cdot V^{-} \cos (\delta-2 \pi / 3)}
\end{gathered}
$$

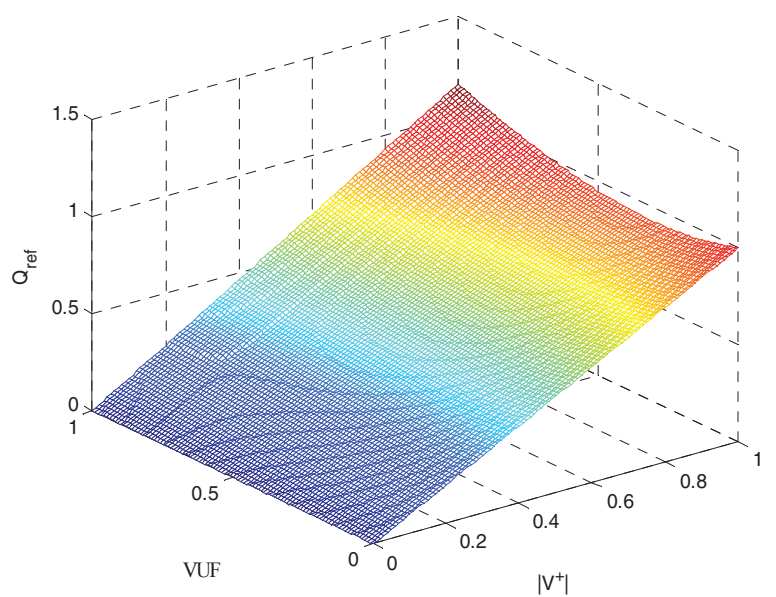

Fig. 4. Maximum permissible reactive current set point in AARC strategy

Combining (23) with (47)-(49) results in Fig. 5 which presents the drop of reference reactive power as a function of voltage unbalanced condition for PNSC strategy.

For a similar amount of voltage dip $\left(V^{+}=0.8 P U\right)$, Fig. 6 visually has compared the maximum permissible reactive power for aforementioned three strategies.

It is clear that in case of PNSC strategy, as the VUF increases the average reactive power descends in order to keep the phase current in a safe band. In contrast, as BPSC strategy does not care about VUF, it decreases the reactive power proportional to the positive sequence voltage. In case of AARC the drop of reference power is more than BPSC in low VUFs but for severe VUFs the average reference reactive power is higher for AARC.

\section{Overall Control Scheme}

The overall control system is built up with the aggregation of voltage limitation and safe current injection limitation as a unified controller that not only cares about peak current limitation but also DC voltage oscillations as well. A simplified block diagram of the proposed control strategy is shown in Fig. 7. A voltage sequence extraction block based on Double Second Order Generalized Integrator (DSOGI) accompanied by a Frequency Locked Loop (FLL) presented in [17] is responsible for the positive and negative sequence voltage extraction in stationary reference frame.

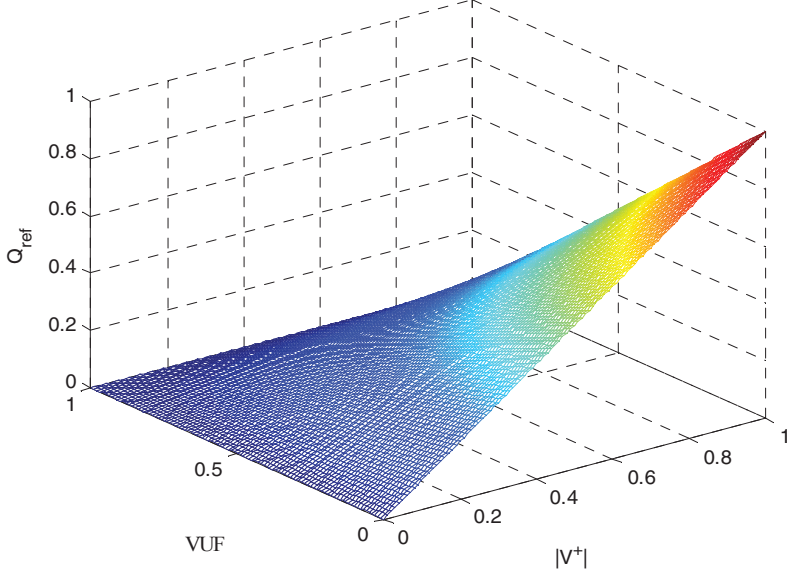

Fig. 5. Maximum permissible reactive current set point in PNSC strategy 


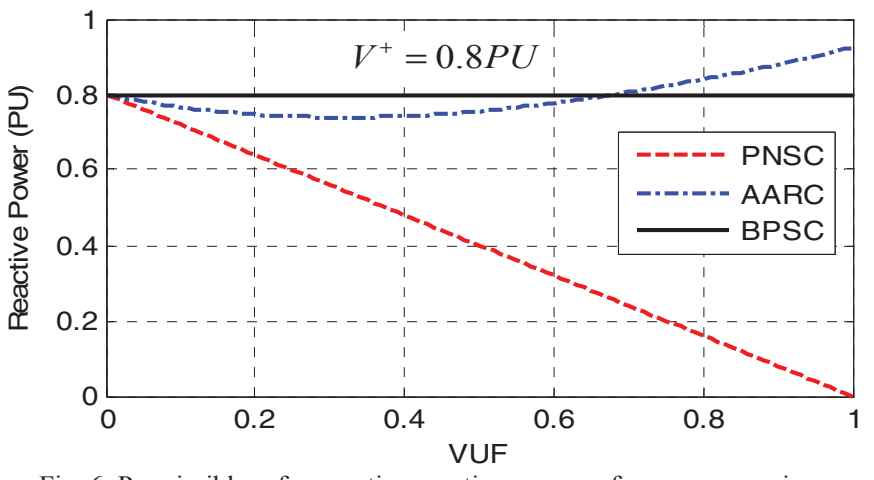

Fig. 6. Permissible safe operating reactive power reference comparison

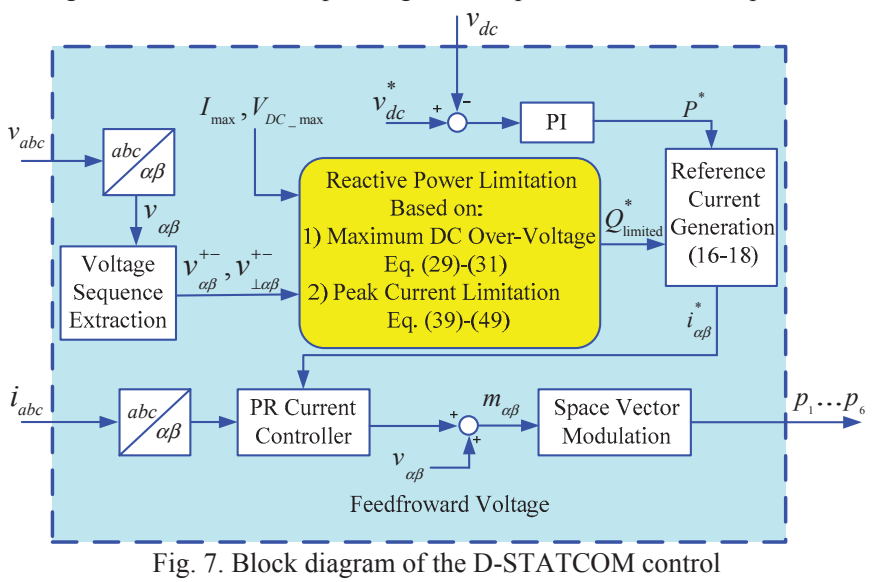

The DC voltage of the capacitor is kept on its nominal average value via a DC voltage control loop. For a fast and accurate tracking of the generated reference currents a couple of Proportional-Resonant (PR) controllers as well as a feedforward voltage from the point of common coupling (PCC) is embedded in the controller. Space Vector Modulation (SVM) is utilized to generate the gating pulses of the switches in a two level Inverter.

\section{Performance Simulation of D-STATCOM in a Weak DISTRIBUTION GRID}

To validate the behavior of the proposed control strategy, the operation of a 4MVA D-STATCOM in a weak distribution grid which is shown in Fig. 8, is analyzed. The nominal DC voltage and the capacitance are $1.4 \mathrm{KV}$ and $10 \mathrm{mF}$ respectively. In this study case, a line to line fault happens in the middle of one of the parallel lines. The behavior of DC voltage, active and reactive powers and their maximum deviations for all the three aforementioned control strategies are presented in Fig. 9. As it can be seen, there is a good matching between the analytical calculations shown in Table IV and the oscillations captured in Fig. 9. Maintaining the peak current and the DC voltage in their secure operation regions is introduced in Fig. 10. It can be seen that utilizing a large DC capacitor in a DSTATCOM yields reaching the current limit faster than the overvoltage limit in the DC bus.

\section{EXPERIMENTAL RESULTS}

The proposed control strategies are implemented in dSPACE DS1103 platform and applied to a 5KVA ,400V inverter with a 700V DC bus and DC capacitance of $4.7 \mathrm{mF}$. The switching frequency is chosen to be $10 \mathrm{kHz}$. The performance of the control strategies, considering the DC voltage and phase current limitations, are evaluated facing a D type voltage sag. Using a voltage sag generator a D-type sag with a characteristics of $0.3 \angle-35^{\circ}$ is applied to the terminal of the converter. The voltage sag occurred when the converter was delivering $3 \mathrm{KVAR}$ (7A peak current) to the grid.

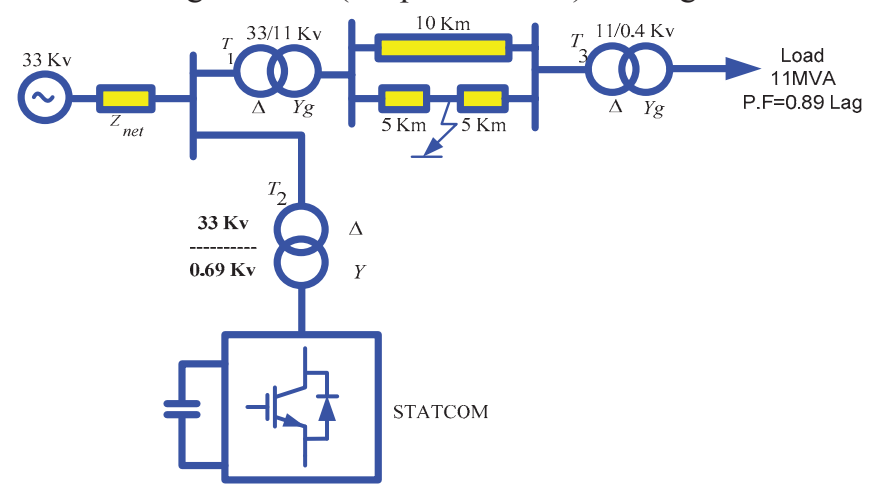

Fig. 8. Connection of a D-STATCOM to a distribution grid
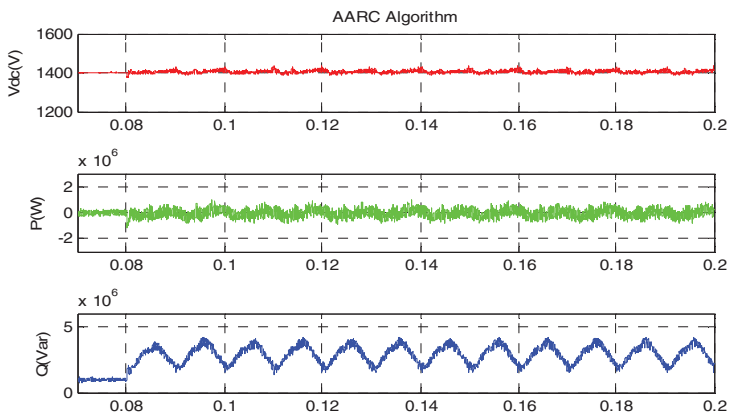

(a)

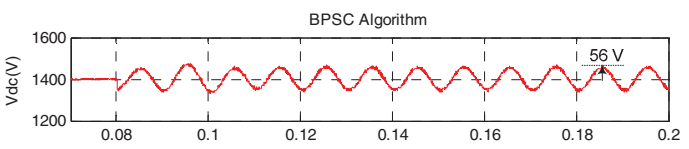

$\times 10^{\circ}$
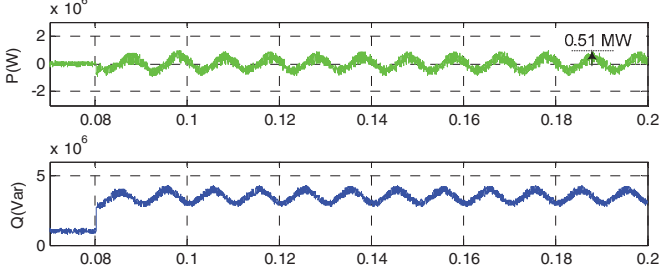

(b)
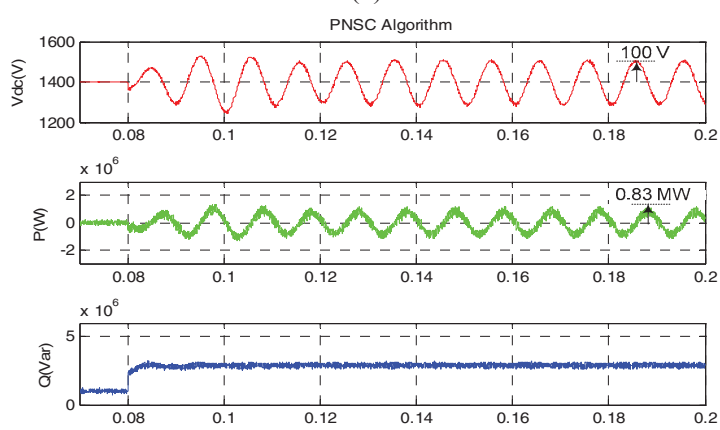

(c)

Fig. 9. DC voltage oscillations and active/reactive powers for a) AARC, b) BPSC, c)PNSC strategy 
Table IV. Analytical expectation for amplitude of active power fluctuations and DC voltage oscillations

\begin{tabular}{|c|c|c|c|}
\hline & AARC & BPSC & PNSC \\
\hline$|\tilde{p}(t)|$ & 0 & $0.486 \mathrm{MW}$ & $0.825 \mathrm{MW}$ \\
\hline$\left|\tilde{v}_{c}(t)\right|$ & 0 & $55 \mathrm{~V}$ & $95 \mathrm{~V}$ \\
\hline
\end{tabular}
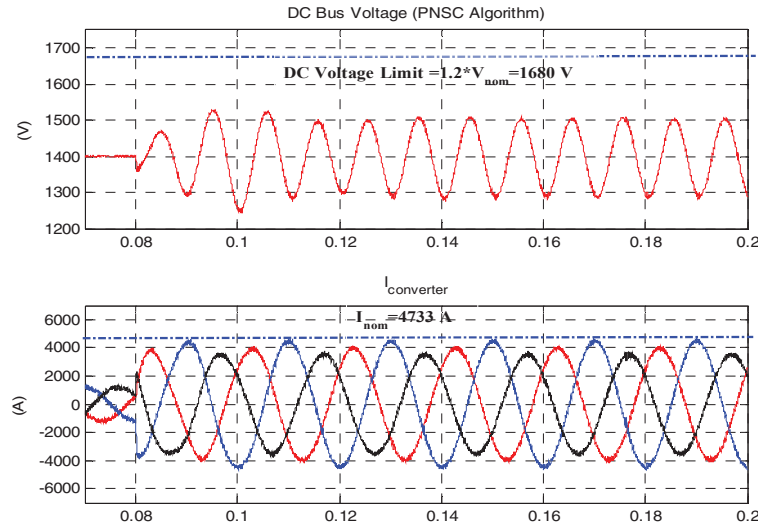

Fig. 10. DC voltage and phase currents are kept in a secure range (PNSC)

Fig .11 shows the unbalanced voltage and the injected currents when using the AARC strategy and Fig .12 is presenting the active power, reactive power as well as DC voltage oscillations in this strategy. During the fault, the phase which experiences more dip has the maximum current and current peaks do not surpass the maximum set point ( $7 \mathrm{~A}$ here). There is no fluctuation in active power and no oscillation in DC voltage either. The reference reactive power decreased from $3 \mathrm{KVA}$ to $1.7 \mathrm{KVA}$ which is superimposed by a $100 \mathrm{~Hz}$ oscillations. Fig. 13 and Fig. 14 are belonging to BPSC strategy. During the unbalanced voltage condition, phase currents are balanced and are limited to the rated current but as a consequence double frequency oscillations appeared in both active and reactive powers. The behavior of PNSC strategy is shown in Fig. 15 and Fig .16. As it can be seen, again currents are bounded in a safe region. In this strategy for the sake of oscillation cancellation in the reactive power, the reference currents are determined in such a way that the phase with lowest voltage dip sinks the most current.

Analyzing the DC voltage oscillations for all three strategies, it can be seen that they meet the expectations inspired by Fig. 3. DC voltage oscillations are almost eliminated in AARC and are the most significant in PNSC.
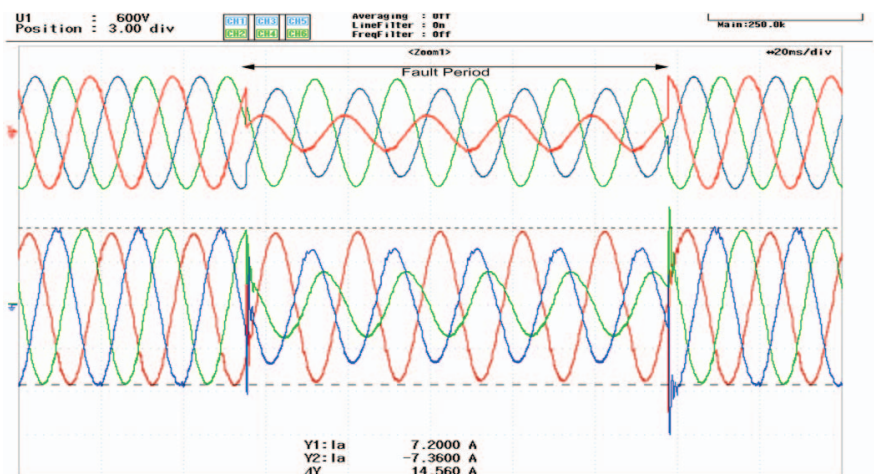

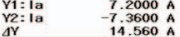

Fig. 11. PCC voltage and injected currents in AARC strategy
On the other side, the values of reactive power reference in Fig. 12, Fig. 14 and Fig. 16 are in good agreement with Fig. 6. It could be deduced that among these three reviewed strategies, BPSC is the best in keeping the reference power as higher as possible. From this insight, AARC treats very close to BPSC but the reference reactive power in PNSC descends drastically as an unbalanced voltage condition occurs.

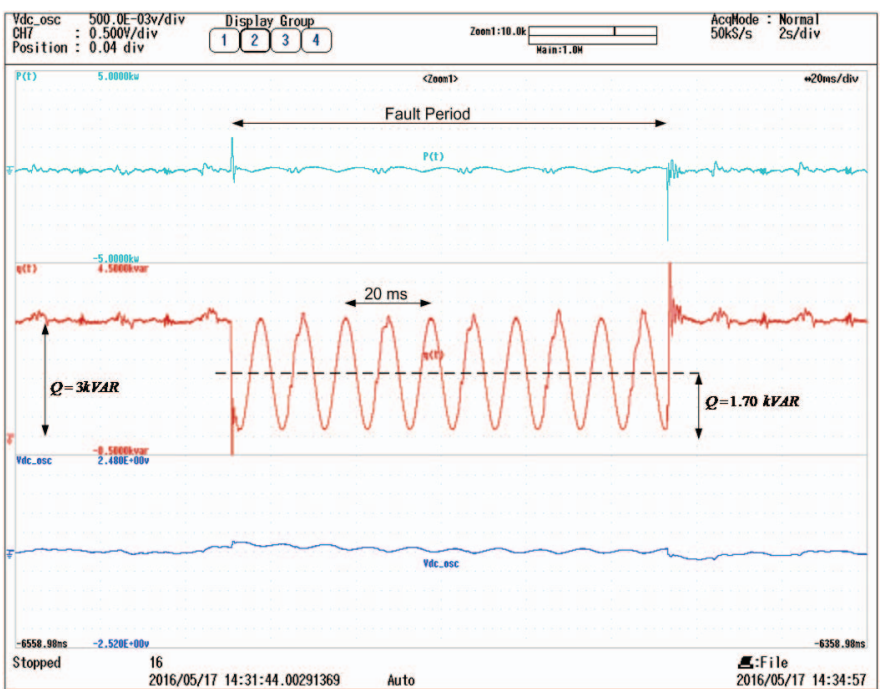

Fig. 12. Active / Reactive power and DC voltage oscillations in AARC

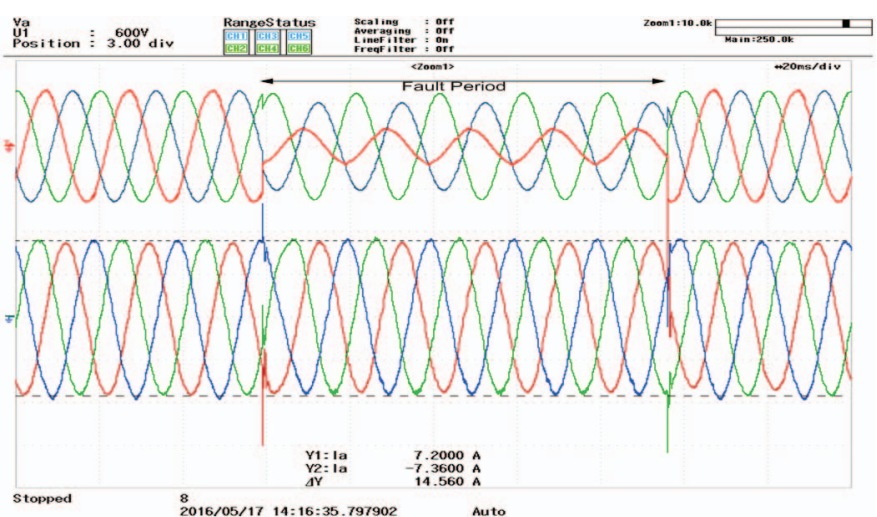

Fig. 13. PCC voltage and injected currents in BPSC strategy

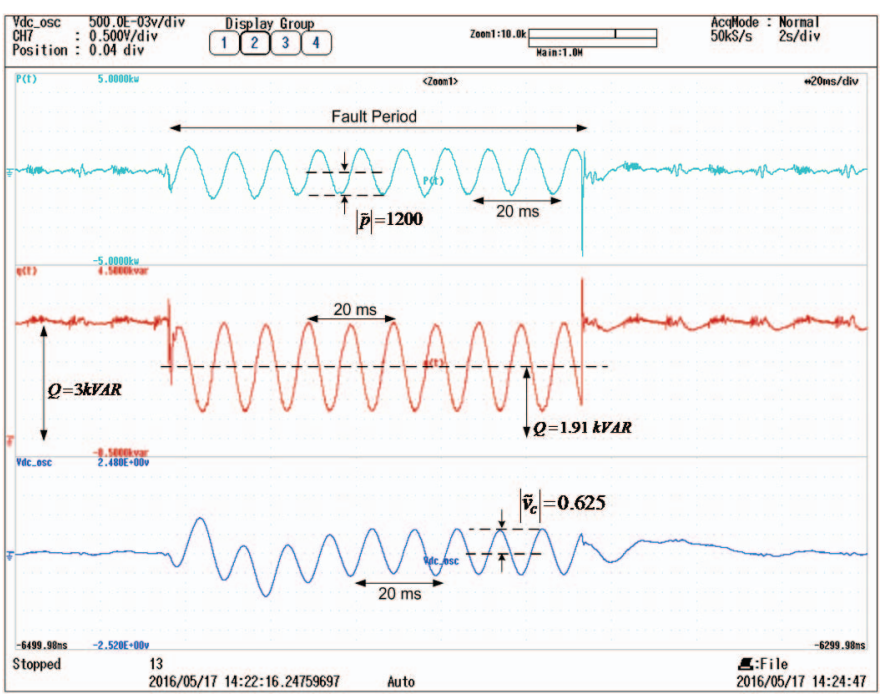

Fig. 14. Active / Reactive power and DC voltage oscillations in BPSC 


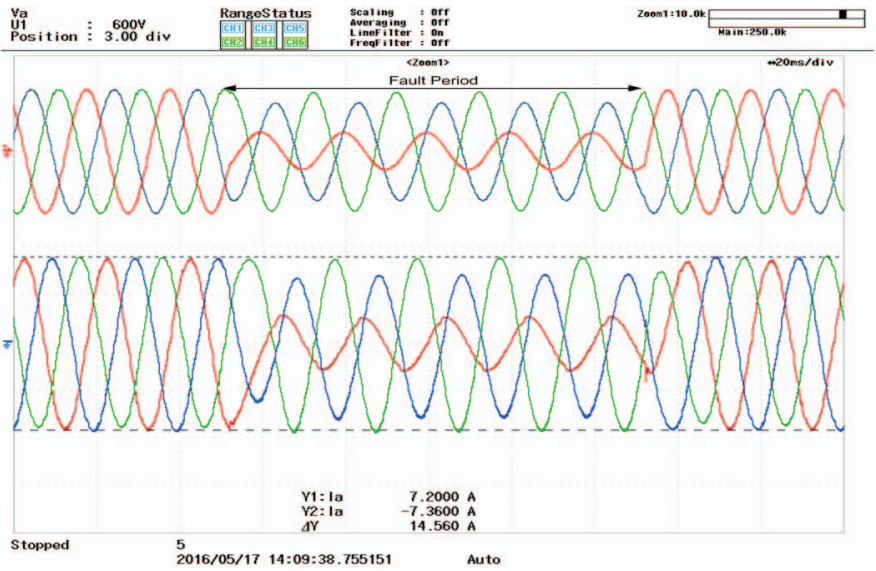

Fig. 15. PCC voltage and injected currents in PNSC strategy

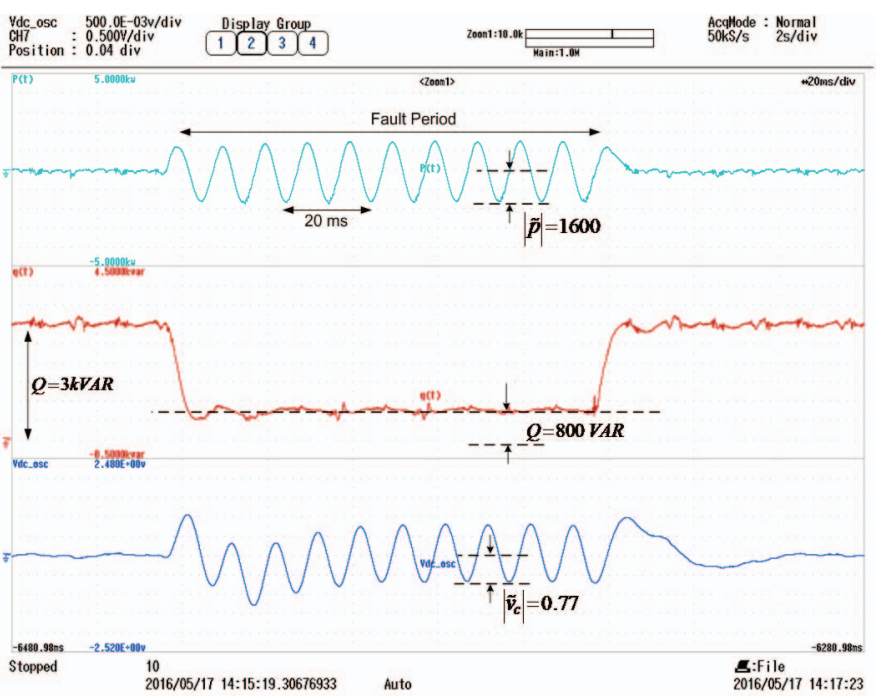

Fig. 16. Active / Reactive powers and DC voltage oscillations in PNSC

Considering both objectives of minimum DC voltage oscillations as well as maximum reactive power delivery, AARC is recognized as the best.

\section{VIII.CONCLUSION}

In this work, safe operation of grid connected converters with regards to peak current limitation as well as maximum permissible DC voltage oscillations. The main effort is concentrated on analyzing of the DC voltage oscillations. Toward this goal, a set of mathematical expressions is developed which prepares a good insight to active power fluctuations (which results in the DC voltage oscillations) as well as maximum current limitation for three different strategies. Deduced analytical expressions are validate by simulations as well as experimental tests and there is a good agreement between them.

\section{ACKNOWLEDGEMENT}

This work has been partially supported by the Spanish Ministry of Innovation and Competitiveness under the projects ENE2013-48428-C2-2-R and ENE2014-60228-R and Iranian Ministry of Science, Research and Technology.

\section{REFERENCES}

[1] "Grid Code. High and extra high voltage", E.ON Netz GmbH, Germany, Apr. 2006.

[2] P. Rodriguez, A. Timbus, R. Teodorescu, M. Liserre, and F. Blaabjerg, "Flexible active power control of distributed power generation systems during grid faults," IEEE Trans. Ind. Electron., vol. 54, no. 5, pp. 25832592 , Oct. 2007

[3] P. Rodriguez, A. Timbus, R. Teodorescu, M. Liserre, and F. Blaabjerg, "Reactive power control for improving wind turbine system behavior under grid faults," IEEE Trans. Power Electron., vol. 24, no. 7, pp. 1798- 1801, Jul. 2009.

[4] F. Wang, J . L. Duarte, and M. A. M. Hendrix, "Pliant active and reactive power control for grid-interactive converters under unbalanced voltage dips," IEEE Trans. Power Electron., vol. 26, no. 5, pp. 15111521, May 2011.

[5] C. Wessels, N. Hoffmann, M. Molinas, F. W. Fuchs, "Statcom control at wind farms with fixed-speed induction generators under asymmetrical grid faults", IEEE Trans. on Ind. Electron., vol. 60, no. 7, pp. 28642873, July 2013

[6] L.Kuang, L. Jinjun, W. Zhaoan, W. Biao, "Strategies and Operating Point Optimization of STATCOM Control for Voltage Unbalance Mitigation in Three-Phase Three-Wire Systems," IEEE Trans. on Power Delivery, vol. 22, no. 1, pp. 413-422, Jan. 2007.

[7] T. Lee, S. Hu, and Y. Chan, "D-STATCOM with positive-sequence admittance and negative-sequence conductance to mitigate voltage fluctuations in high-level penetration of distributed generation systems," IEEE Trans. Ind. Electron., vol. 60, no. 4, pp. 1417-1428, Apr. 2013.

[8] Castilla, M.; Miret, J.; Camacho, A.; Matas, J.; Garcia de Vicuna, L. "Voltage Support Control Strategies for Static Synchronous Compensators Under Unbalanced Voltage Sags", IEEE Trans. Ind. Electron, vol. 61, no. 2, pp. 808-820, Feb. 2014

[9] P. Rodriguez, G. Medeiros, A. Luna, M. C. Cavalcanti, and R. Teodorescu, "Safe current injection strategies for a STATCOM under asymmetrical grid faults," in Proc. IEEE ECCE, 2010, pp. 3929-3935.

[10] M. T. Bina and M. D. Eskandari, "Consequence of unbalance supplying condition on a distribution static compensator," in Proc. IEEE 35th Annu.Power Electron. Spec. Conf., 2004, vol. 5, pp. 3900-3904.

[11] S. Babaei, B. Fardanesh, S.Bhattacharya, "High-Power VSC-based simultaneous positive and negative sequence voltage regulator", IEEE Trans. Power Del., vol. 29, no. 5, pp. 2124-2135, Oct. 2014

[12] R. Teodorescu, M. Liserre, and P. Rodriguez, Grid Converters for Photovoltaic and Wind Power Systems. , USA: John Wiley \& Sons, 2011,ch.10,pp.237-288.

[13] H. Akagi, E. H. Watanabe, and M. Arades, Instantaneous Power Theory and Applications to Power Conditioning, NJ, USA: John Wiley \& Sons, 2007.

[14] A.Luna, J.Rocabert, I.Candela, J.Hermoso, R.Teodorescu, F.Blaabjerg, P.Rodriquez, "Grid voltage synchronization for distributed generation systems under grid fault conditions,"IEEE Trans. Ind. Appl. Vol.51, no. 4, pp. 3414-3425, July/August 2015.

[15] M. Mishra and K. Karthikeyan, "A fast-acting DC-link voltage controller for three-phase DSTATCOM to compensate AC and DC loads," IEEE Trans. Power Del., vol. 24, no. 4, pp. 2291-2299, Oct. 2009.

[16] B. Singh, A. Chandra, and K. Al-haddad, Power Quality Problems and Mitigation Techniques, UK : John Wiley \& Sons, 2015.

[17] P. Rodriguez, A.Luna, I.Candela, R. Mujal, R. Teodorescu, and F. Blaabjerg, "Multiresonant frequency-locked loop for grid synchronization of power converters under distorted grid conditions," IEEE Trans.Ind. Electron., vol. 58, no. 1, pp. 127-138, Jan. 2011. 